This is an emulation of Solar Physics $\bullet \bullet \bullet \bullet \bullet-\bullet \bullet \bullet, ~ 2009$

The authoritative version is DOI: $10.1007 /$ s11207-009-9399-5

\title{
Lifetimes of High-Degree $p$ Modes in the Quiet and Active Sun
}

\author{
O. Burtseva ${ }^{1}$ F. Hill ${ }^{1}$ S. Kholikov ${ }^{1}$ \\ D.-Y. $\mathrm{Chou}^{2}$
}

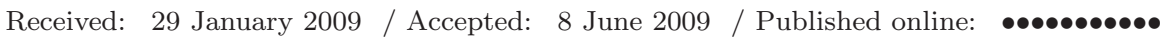

\begin{abstract}
We study variations of the lifetimes of high- $\ell$ solar $p$ modes in the quiet and active Sun with the solar activity cycle. The lifetimes in the degree range $\ell=$ $300-600$ and $\nu=2.5-4.5 \mathrm{mHz}$ were computed from $\mathrm{SOHO} / \mathrm{MDI}$ data in an area including active regions and quiet Sun using the time-distance technique. We applied our analysis to the data in four different phases of solar activity: 1996 (at minimum), 1998 (rising phase), 2000 (at maximum), and 2003 (declining phase). The results from the area with active regions show that the lifetime decreases as activity increases. The maximal lifetime variations are between solar minimum in 1996 and maximum in 2000; the relative variation averaged over all $\ell$ values and frequencies is a decrease of about $13 \%$. The lifetime reductions relative to 1996 are about $7 \%$ in 1998 and about $10 \%$ in 2003 . The lifetime computed in the quiet region still decreases with solar activity although the decrease is smaller. On average, relative to 1996, the lifetime decrease is about $4 \%$ in $1998,10 \%$ in 2000 , and $8 \%$ in 2003 . Thus, measured lifetime increases when regions of high magnetic activity are avoided. Moreover, the lifetime computed in quiet regions also shows variations with the activity cycle.
\end{abstract}

Keywords: Sun: helioseismology, time-distance, $p$-mode lifetime, solar activity

\section{Introduction}

The excitation of solar acoustic oscillations is attributed to turbulent convection beneath the solar surface. The structural and dynamical properties of the Sun, as well as the processes of excitation and damping of the acoustic modes, change with the solar cycle. Variation in the excitation and damping are observed as changes in the mode amplitudes and lifetimes. The study of variations in these parameters provides information on the mechanism driving the solar oscillations.

\footnotetext{
${ }^{1}$ National Solar Observatory, 950 North Cherry Ave., Tucson, AZ 85719, USA (e-mail: burtseva@noao.edu)

${ }_{2}$ Institute of Astronomy and Department of Physics, Tsing

Hua University, Hsinchu, 30043, Taiwan
} 
The $p$-mode lifetime in global helioseismology is computed from the line width as $(2 \pi \gamma)^{-1}$, where $\gamma$ is the FWHM of the $p$-mode line profile in power spectra. Several authors (Chaplin et al., 2000; Jimenez-Reyes et al., 2003: Howe et al., 2003) have shown significant temporal variations in the mode widths using low- $\ell$ data. They reported a $23 \%$ increase in the mode widths (i.e. decrease in the mode lifetimes) with increasing solar activity. They also found that the variations are frequency dependent, being largest near $3.1 \mathrm{mHz}$, and independent of $\ell$. The results have been confirmed with intermediate- $\ell$ data, showing a $14 \%$ increase in the widths with activity (Komm, Howe, and Hill, 2000; Salabert and Jimenez-Reyes, 2006)

The authors also analyzed changes in the mode amplitudes, power, and energysupply rate from solar minimum to maximum and concluded that the variations probably arise from an increase of the damping only, since the net forcing of the modes remains constant. Some of the authors noted that, even if no significant changes in global $p$-mode excitation are evident, it may possibly change locally. Possible mechanisms, in addition to a continuous turbulent source of excitation, are transient phenomena such as flares (Haber et al., 1988, Kosovichev and Zharkova, 1998: Donea, Braun, and Lindsey, 1999: Ambastha, Basu, and Antia, 2003 Martinez-Oliveros, Moradi, and Donea, 2008: Karoff and Kjeldsen, 2008) and CMEs (Gavryusev and Gavryuseva, 1999).

One potential source of damping of the acoustic waves is magnetic activity that is present on the solar surface. Results of global (Komm, Howe, and Hill, 2002) and local (Rajaguru, Basu, and Antia, 2001; Howe et al., 2004) analyses have shown a strong dependence of the mode amplitudes and lifetimes on the locations of active regions, with amplitude and lifetime decreasing in the presence of strong magnetic fields. Also, in contrast to the global analysis, Howe et al. (2004) reported a significant decrease of the energy supply rate of $p$ modes in active regions. It is known that the power of the oscillations in sunspots and plages is twothree times lower than in the quiet Sun (Woods and Cram, 1981). Possible mechanisms that might be responsible are the suppression of excitation in sunspots as a strong magnetic field inhibits convection (Parchevsky and Kosovichev, 2007), absorption of the $p$-mode waves in sunspots (Braun, Duvall, and LaBonte, 1987; Chen et al., 1996; Chou et al., 1996), the different height of spectral-line formation due to the Wilson depression, and the modification of $p$-mode eigenfunctions by the magnetic field (Hindman, Jain, and Zweibel, 1997).

Another possible contribution to $p$-mode damping is variations in the convective properties near the solar surface. Most likely, these variations arise from the influence of magnetic structures, as thermal perturbations in the convection zone cannot alone explain observed changes in irradiance and $p$-mode frequencies over the activity cycle (Balmforth, Gough, and Merryfield, 1996). Houdek et al. (2001) made an attempt to connect changes in the horizontal length scale of convective eddies and the $p$-mode damping rates and found that decreasing the horizontal size of convective eddies increases the $p$-mode damping.

Whatever mechanisms generate and damp solar $p$ modes and drive the solar cycle, they are believed to operate in the convection zone. High-degree $(\ell>200)$ acoustic waves propagate in the upper convection zone and yield information about inhomogeneities in structure and dynamics of this region. Determination of the line widths and other parameters of the high- $\ell p$ modes has been a problem 
because of the blending of different modes in power spectra. Burtseva et al. (2007) compared the lifetime of high- $\ell p$ modes at solar minimum and maximum using an alternative method based on time-distance analysis first proposed by Chou et al. (2001). The conclusion of Burtseva et al. (2007) is consistent with low- and intermediate- $\ell$ analysis (i.e., the lifetime decreases with increasing solar activity). In this work we use the same method to measure lifetimes of highdegree solar $p$ modes in the range $\ell=300-600$ and $\nu=2.5-4.5 \mathrm{mHz}$ in four different phases of solar cycle in an area including active regions and a quiet-Sun area and discuss the results.

\section{Method of Lifetime Measurement}

The lifetime measured with time-distance analysis is the lifetime of a wave packet, not of an individual mode. The wave-packet lifetime is determined by changes in the amplitude and width of the cross-correlation function of the packet with time. The amplitude of the cross-correlation function decreases exponentially with the number of skips of the wave below the photosphere. This effect has been interpreted as the dissipation of solar $p$-mode power (Chou et al., 2001). As a wave packet consists of many $p$ modes over some range of phase velocities $(2 \pi \nu / \ell)$ dispersion causes it to extend (i.e., its amplitude decreases and its width increases). In previous studies (Chou and Ladenkov, 2007; Burtseva et al., 2007), it was found that the width of the cross-correlation function increases with the number of skips, demonstrating the effect of dispersion of the wave packet. Dispersion does not change the energy of the wave packet, which is the product of amplitude squared and width of the wave packet (Jackson, 1975). Taking into account both the dissipation and the dispersion, we can define the lifetime as the e-folding time of the energy of the wave packet due to dissipation (Chou and Ladenkov, 2007; Burtseva et al., 2007):

$$
A_{n}^{2} W_{n}=A_{0}^{2} W_{0} e^{-n \tau_{e n} / T},
$$

where $A_{n}$ and $W_{n}$ are the amplitude and the width of the cross-correlation of skip $n$, respectively, $\tau_{\text {en }}$ is the one-skip envelope time, and $T$ is the lifetime of the wave packet.

\section{Data Analysis}

In this study we analyzed two 512-minute series of MDI Dopplergrams in each of the following epoch: at solar minimum (24 May and 18 September 1996), in the rising phase of activity ( 5 and 6 February 1998), at solar maximum (9 and 11 September 2000), and in the declining phase of activity (12 and 13 December 2003).

We obtained information on the distribution of magnetic features and the magnetic-field strength on the analyzed days using MDI 96-minute magnetograms and acoustic-power maps. The areas including active regions and the quiet areas 
Table 1. Unsigned magnetic field (in Gauss) averaged over the whole area including active regions and the quiet area (see Figure 1).

\begin{tabular}{lcccc}
\hline Date & $\begin{array}{c}\text { Area including } \\
\text { active regions } \\
(\mathrm{MDI})\end{array}$ & $\begin{array}{c}\text { Quiet Area } \\
(\mathrm{MDI})\end{array}$ & $\begin{array}{c}\text { Area including } \\
\text { active regions } \\
(\mathrm{KPM})\end{array}$ & $\begin{array}{c}\text { Quiet Area } \\
(\mathrm{KPM})\end{array}$ \\
\hline 24 May 1996 & - & - & $1.73 \pm 0.03$ & $2.49 \pm 0.11$ \\
18 Sep 1996 & $2.43 \pm 0.02$ & $1.90 \pm 0.07$ & $1.98 \pm 0.03$ & $2.80 \pm 0.11$ \\
05 Feb 1998 & $3.60 \pm 0.04$ & $1.71 \pm 0.04$ & $3.25 \pm 0.07$ & $2.15 \pm 0.11$ \\
06 Feb 1998 & $4.45 \pm 0.06$ & $2.02 \pm 0.07$ & $3.01 \pm 0.08$ & $2.05 \pm 0.13$ \\
09 Sep 2000 & $15.03 \pm 0.33$ & $5.40 \pm 0.12$ & $15.00 \pm 0.12$ & $6.75 \pm 0.20$ \\
11 Sep 2000 & $16.21 \pm 0.33$ & $4.93 \pm 0.17$ & $15.20 \pm 0.11$ & $5.42 \pm 0.16$ \\
12 Dec 2003 & $3.99 \pm 0.26$ & $3.37 \pm 0.14$ & - & - \\
13 Dec 2003 & $5.85 \pm 0.27$ & $3.53 \pm 0.14$ & - & - \\
\hline
\end{tabular}

analyzed in this work are shown in Figure 1. Concentrations of active regions are denoted with black contours, which correspond to the magnetic field level of $25 \mathrm{G}$ after smoothing of the images. It is seen that there is no concentration of active regions in the quiet areas. On both days in 1996 there were no active regions present on the solar disk. On the days in 2000 the Sun was active with extended active regions, with a relatively low maximal value of magnetic field of 800 G. On the days in 1998 and 2003 the central part of the Sun was quiet, but a few small sunspots with magnetic field up to $2000 \mathrm{G}$ were present on the disk at about $40^{\circ}$ from the disk center.

We characterize the activity level on the analyzed days by computing a magnetic activity index (MAI). The $1 \sigma$ noise level in a single MDI magnetogram is about $20 \mathrm{G}$ (Scherrer et al., 1995). To reliably compute MAI we first set all unsigned flux less than $2.5 \sigma=50 \mathrm{G}$ to zero and correct images for outliers using a technique similar to that described in (Basu, Antia, and Bogart, 2004). We compute MAI by summing the absolute values of the magnetic flux inside the analyzed areas and normalizing the sum by the total number of pixels in the areas instead of the number of nonzero pixels. We also compute MAI using NSO/Kitt Peak magnetograms (KPM) for comparison. The $1 \sigma$ noise level of KPM data is about $5 \mathrm{G}$ (Wenzler et al., 2004). Similarly, we set all unsigned flux less than $2.5 \sigma=12 \mathrm{G}$ to zero. Then we multiply the KPM images by 1.7 to correct for the difference in scale as MDI shows a larger magnetic flux than KPM. The computed MAIs are shown in Table 1. The MDI magnetograms for 24 May 1996 and KPM data for the two days in 2003 are absent.

The data analysis and cross-correlation function computation procedure is similar to that used in (Burtseva et al., 2007) and briefly described as follows:

i) Images are remapped, tracked, filtered with 15-minute running mean, and transformed into the $(\ell, m, \nu)$ domain, where $m$ is the azimuthal degree.

ii $\quad$ A Gaussian filter of FWHM $=2.0 \mathrm{mHz}$ centered at a frequency $\nu_{0}$ is applied.

iii) A phase-velocity filter is applied to isolate the modes in a range of angular phase velocity $(w=2 \pi \nu / \ell)$ forming a wave packet with the central fre- 


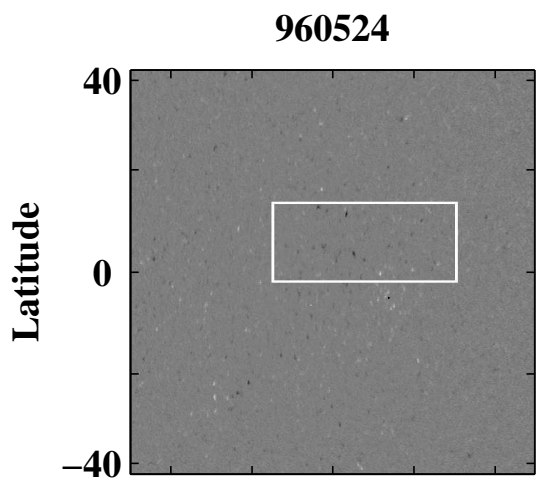

960918
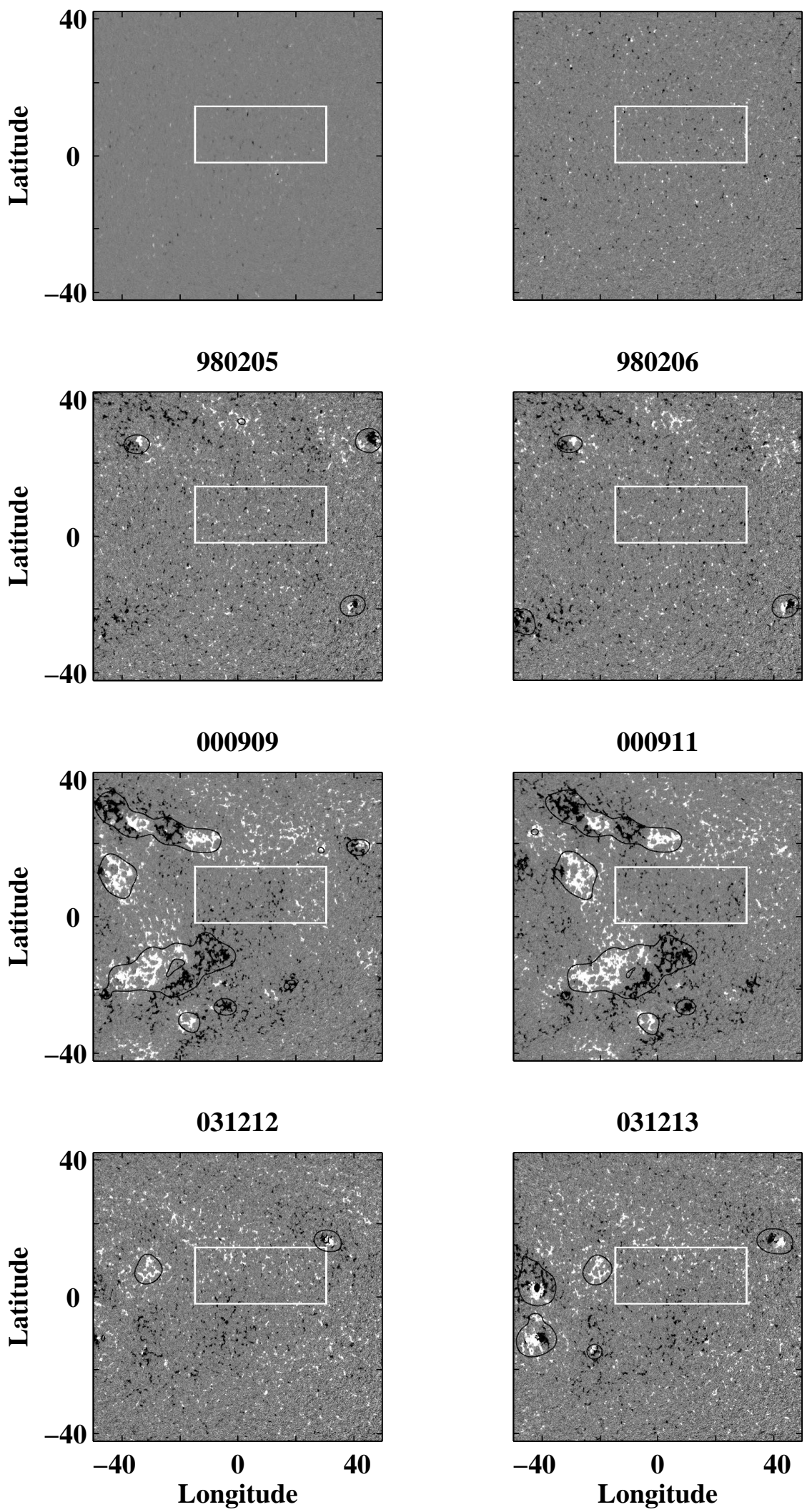

Figure 1. MDI magnetograms of the analyzed days. Lifetimes were computed using the whole area $\left(99.4^{\circ} \times 85.2^{\circ}\right)$, including active regions, and in the quiet area $\left(45.9^{\circ} \times 15.7^{\circ}\right)$ shown as a white box around the disk center. Black contours denote active-region concentrations. 
quency $\nu_{0}$ and the corresponding degree $\ell_{0}$. The filter is smoothed with a Hanning window in frequency.

$i v$ ) The data are reconstructed back to the space-time domain.

$v$ ) The center-annuli cross-correlation $C(\Delta, \tau)$ is computed with

$$
C(\Delta, \tau)=\int \Psi(0, t) \Psi(\Delta, t+\tau) \mathrm{dt},
$$

where $\Psi(0, t)$ is the signal measured at the central point at time $t$ and $\Psi(\Delta, t+\tau)$ is the signal averaged over an annulus at an angular distance $\Delta$ from the central point at time $t+\tau$. The procedure is repeated for different central points at the disk center. The cross-correlation functions of different central points are averaged.

vi) For each skip, $C(\Delta, \tau)$ is fitted by a Gabor wavelet (Kosovichev and Duvall, 1997)

$$
G(\tau)=A \cdot \cos \left[2 \pi \nu\left(\tau-\tau_{p h}\right)\right] \cdot \exp \left[-\frac{\left(\tau-\tau_{e n}\right)^{2}}{2 W^{2}}\right],
$$

where $A, \nu$, and $W$ are the amplitude, frequency, and width of a Gaussian envelope, respectively. The parameters $\tau_{\mathrm{ph}}$ and $\tau_{\mathrm{en}}$ are the phase time and envelope time, respectively. For each skip, $A, W$, and $\tau_{\text {en }}$ are averaged over five angular distances near the maximum of the cross-correlation amplitude to reduce noise. The procedure is repeated for both positive and negative $\tau$. The values of $A$ and $W$ are the averages of results for positive and negative $\tau$, since they are found to be very close in magnitude.

vii) The lifetime $(T)$ is determined by fitting $\ln \left(A^{2} W\right)$ versus $n$ according to Equation (1). The details are discussed in Burtseva et al. (2007).

The above analysis was performed for the whole area including active regions and the quiet area near the disk center shown in Figure 1. A minimum fourskip distance is required to compute lifetimes with this method. The size of the quiet area does not allow us to perform the analysis for the wave packets with $\ell_{0}=300, \nu_{0}=2.5-4.5 \mathrm{mHz}$ and $\ell_{0}=400, \nu_{0}=4.0$ and $4.5 \mathrm{mHz}$ as the distance required for the lifetime computation for those waves is from about $13^{\circ}$ to $24^{\circ}$. For the quiet area we have about $7^{\circ}$ maximum, thus we are able to compute lifetimes only for the wave packets $\ell_{0}=400, \nu_{0}=2.5-3.5 \mathrm{mHz} \ell_{0}=500-600$, $\nu_{0}=2.5-4.5 \mathrm{mHz}$ which requires distances from about $5^{\circ}$ to $7^{\circ}$.

\section{Results and Discussion}

The lifetimes of solar $p$ modes computed in the area including active regions are shown in Figure 2. It is seen that the lifetime decreases as activity increases. The results are in agreement with all previous studies of the $p$-mode lifetimes mentioned earlier. The percentage of the lifetime decrease for all analyzed days relative to the days in 1996 is listed in Table 2 . The maximal lifetime variations are between solar minimum in 1996 and maximum in 2000; the relative variation averaged over all $\ell$ values and frequencies is about 13\%. The lifetimes in 2003 

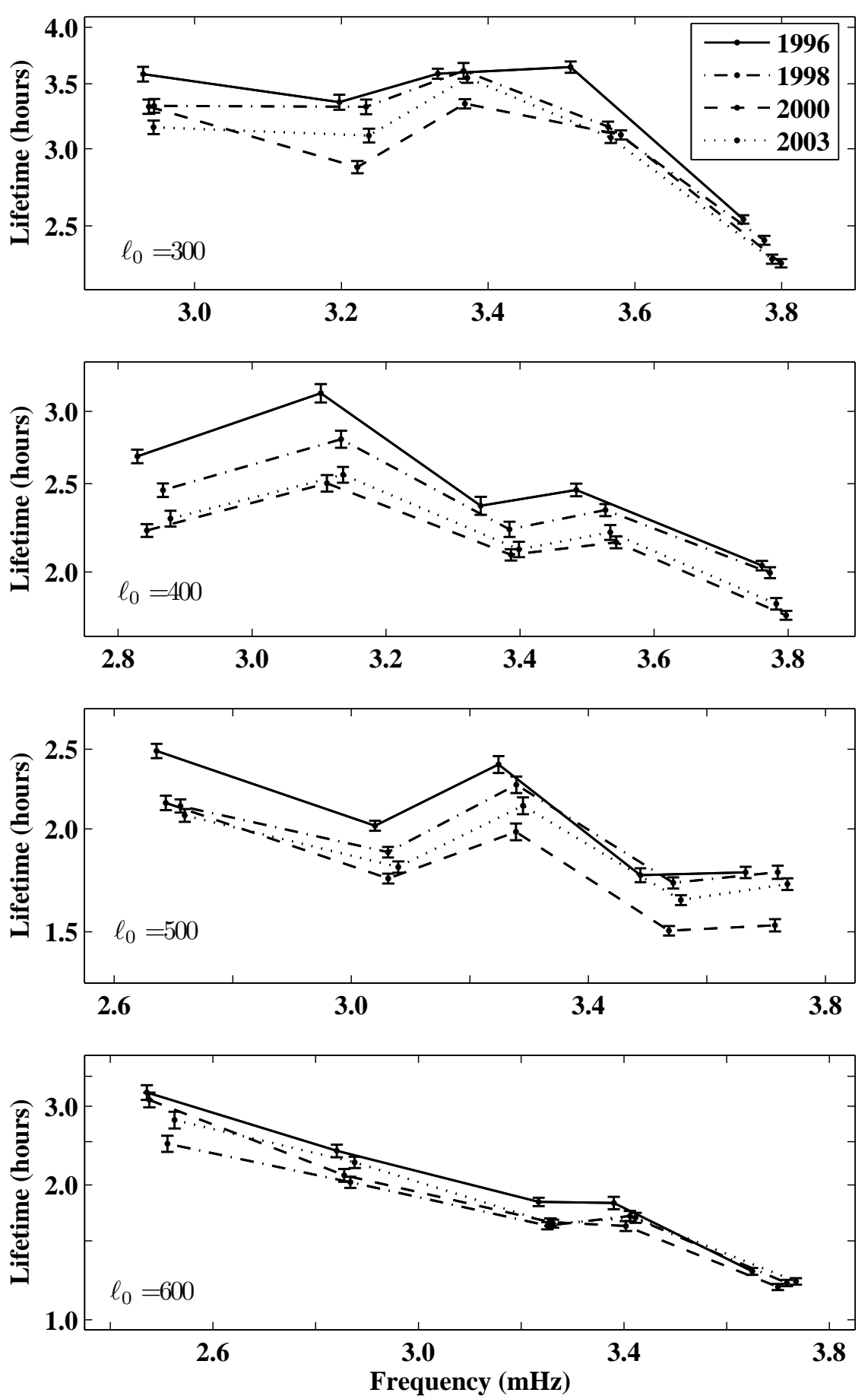

Figure 2. $p$-mode lifetime computed in the area including active regions, averaged over the results from two 512-minute time series in each epoch versus frequency for various $\ell_{0}$. The frequency is determined from the Gabor fit for the first-skip cross-correlation function. 

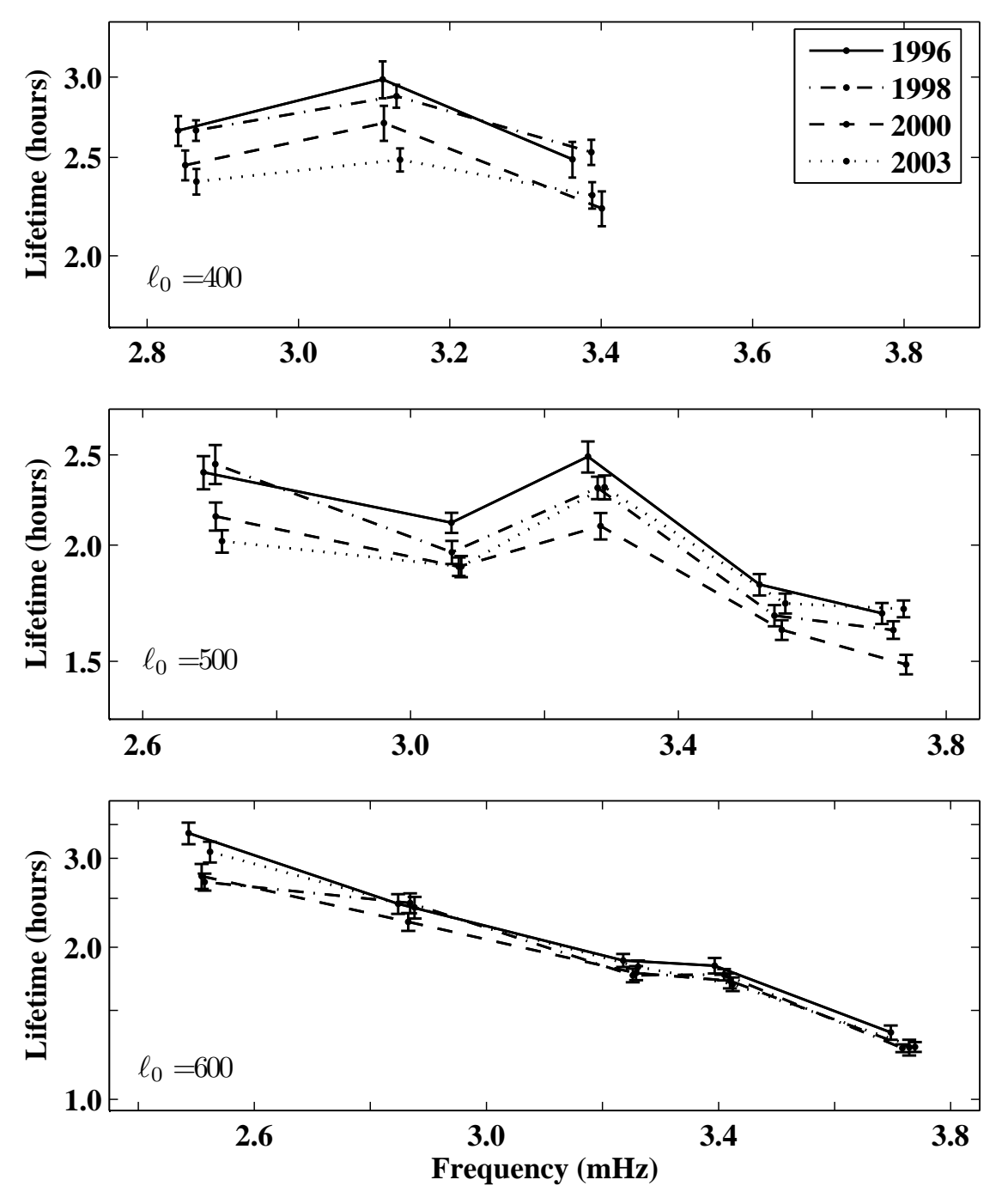

Figure 3. $p$-mode lifetime computed in the quiet area, averaged over the results from two 512 -minute time series in each epoch versus frequency for various $\ell_{0}$. The frequency is determined from the Gabor fit for the first-skip cross-correlation function.

are closer to the lifetimes in 2000, and the lifetimes in 1998 are closer to those in 1996. The lifetime variations relative to 1996 are about $7 \%$ in 1998 and about $10 \%$ in 2003. As seen in Table 1, the activity level was higher on the analyzed days in 2003 than in 1998.

The lifetimes computed in the quiet area are presented in Figure 3. After excluding active regions from the analysis, the lifetimes decrease with solar activity, although the decrease is smaller. On average, relative to 1996, the decrease in the lifetime is about $4 \%$ in 1998, 10\% in 2000, and $8 \%$ in 2003. 
The daily variations of the lifetime in 1996 are within the uncertainties of the measurements.

Thus, excluding high magnetic activity regions increases the measured lifetime. From the difference between lifetimes and the magnetic field strength of the quiet area and the area including active regions, and also from the distribution of the active features on the solar disk on the analyzed days (see Figure 1), we speculate that acoustic waves are affected more by large active regions as seen at solar maximum, and less by small sunspots with strong magnetic field. This is likely because the wavelengths of the acoustic waves are typically larger than the smaller sunspots and better matched with the large active regions.

The lifetime computed in the quiet area still shows variations with activity cycle. What could cause the variations in the quiet area where there are no largescale magnetic field concentrations? One explanation could be that the convective properties change with solar cycle. As discussed in the Introduction, Houdek et al. (2001) found that damping of $p$ modes increases as horizontal size of convective eddies decreases. It has been reported by some authors (Muller, 1988, Berrilli et al., 1999) that the horizontal scale of the solar granules decreases from solar minimum to maximum, which perhaps indicates that the horizontal size of the convective eddies does indeed decrease. Meunier et al. (2008) reported that supergranular cells are smaller at solar maximum. However, other studies arrive at the contradictory conclusion that granular size increases with activity (Hanslmeier and Muller, 2002, Saldana-Munoz, Muller, and Hanslmeier, 2004).

Solar-cycle variation of the lifetime in the quiet area caused by purely structural perturbations in the convection zone should result in lifetime variations with no correlation with magnetic field strength. To test this we computed the average lifetime for each wave packet over the four epochs and normalized each of the lifetimes by the average. Then we plot the normalized lifetimes of all wave packets analyzed versus MAI computed in the area including active regions and the quiet area in Figure 4. The lifetimes obviously anticorrelate with MAI for the area including active regions. In the quiet area we also see an inverse correlation of the lifetimes with MAI. This suggests that the magnetic field somehow plays a role in the lifetime variation of $p$ modes in the quiet Sun. Future work will involve more statistics to confirm the conclusions.

All of the analyzed quiet regions have different levels of magnetic field (see Table 1). Some isolated pixels in the quiet regions have magnetic fields as strong as $\approx 500 \mathrm{G}$, which are the strong-field component of the network field at the supergranular boundaries (Harvey, 1971, Lin, 1995).

Table 2. Percentage of the lifetime decrease relative to 1996, averaged over all $\ell$ values and frequencies of $3.0,3.5$, and $4.0 \mathrm{mHz}$.

\begin{tabular}{ccc}
\hline Year/1996 & Area including active regions (\%) & Quiet Area (\%) \\
\hline $1998 / 1996$ & 6.9 & 4.2 \\
$2000 / 1996$ & 13.2 & 9.6 \\
$2003 / 1996$ & 9.6 & 7.6 \\
\hline
\end{tabular}



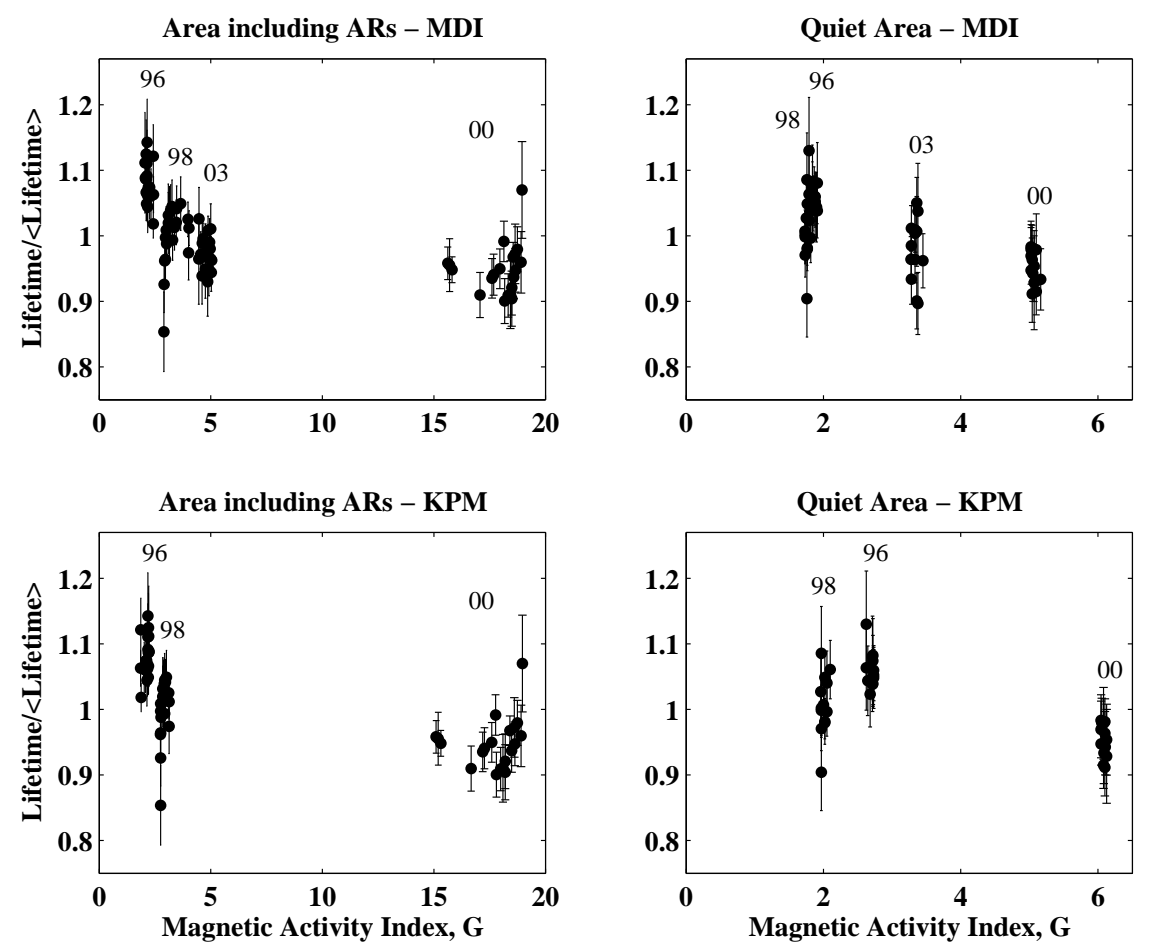

Figure 4. Normalized lifetimes versus magnetic activity index in the area including active regions (left) and the quiet area (right). Top and bottom panels correspond to the MAI computed from MDI and Kitt Peak magnetograms, respectively.

Thus, the elements of heightened magnetic activity in the quiet regions could cause the residual lifetime variations in the quiet area. The MAIs of the analyzed quiet areas also show solar-cycle variations. One can clearly see the increase in MAI from Table 1 (plotted in Figure 5) corresponding to the days at solar maximum for both the area with active regions and the quiet area. Most authors who analyzed solar-cycle variations of the irradiance and photospheric magnetic field reported little overall solar-cycle variations in the quiet-Sun network elements arising from decaying active regions, and no variations in the intranetwork field (Harvey, 1994: Topka, Tarbell, and Title, 1997; Pevtsov and Acton, 2001: Hagenaar et al., 2003 Sanchez Almeida, 2003 Pauluhn and Solanki, 2003). Muller et al. (2007) reported solar-cycle related variations of the granulation contrast at the solar surface connected to the temperature differences between granules and intergranular lanes. The contrast variations may be due to changes in the physical structure under the surface or a varying amount of magnetic flux in the intergranular lanes.

The recently discovered horizontal magnetic field in quiet regions in the solar photosphere (Harvey et al., 2007, Lites et al., 2008) might also affect the propagation of the acoustic waves in the quiet Sun in a way different from that of the vertical component of the magnetic field. As the authors speculate, the possible driving mechanism for the horizontal field is granular and supergranular 


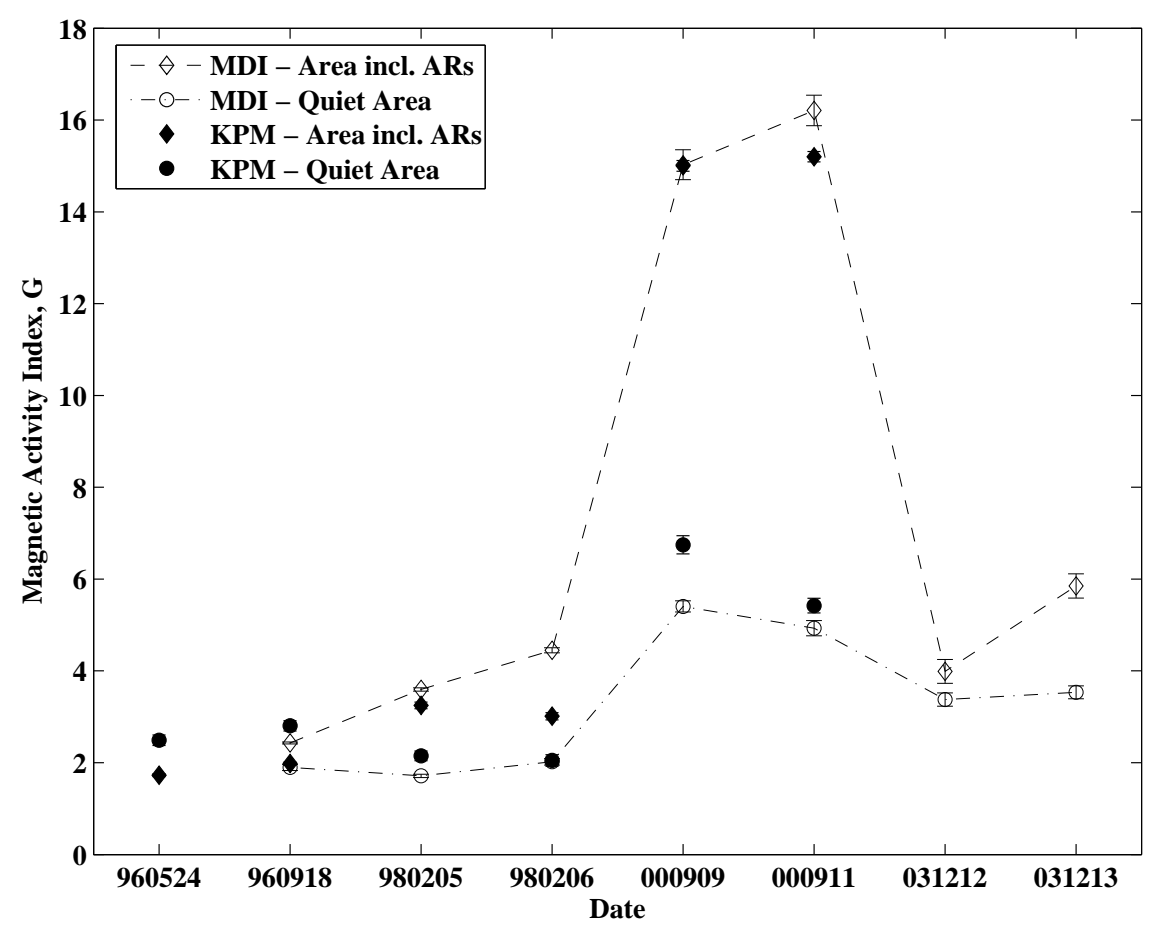

Figure 5. Temporal evolution of MAIs from Table 1 vs. four epochs used in the lifetime study. The line and symbol styles are shown in the top-left corner of the figure. The plot clearly shows solar cycle variations of the MAI in both the area including active regions and the quiet area.

convection and transformation of the field lines in response to the evolving flux distribution in the network and intranetwork field in the quiet Sun.

In future work, we will analyze a large number of quiet areas to confirm temporal variations of the lifetimes of high- $\ell$ acoustic waves found in this work. We will also study variability of the apparent power of the acoustic modes in an area including active regions and a quiet area in different phases of the solar cycle.

\section{Acknowledgments}

The National Solar Observatory is operated by AURA, Inc. under a cooperative agreement with the National Science Foundation. SOHO is a mission of international cooperation between ESA and NASA. NSO/Kitt Peak data used here are produced cooperatively by NSF/NSO, NASA/GSFC, and NOAA/SEL. The authors thank R. Bogart, J. Harvey, C. Lindsey, J.C. Martinez-Oliveros, A. Norton, G. Petrie, N.-E. Raouafi, and S. Tripathy for fruitful discussions of the results of this work, helpful suggestions, and comments. 


\section{References}

Ambastha, A., Basu, S., Antia, H.M.: 2003, Solar Phys., 218, 151.

Balmforth, N.J., Gough, D.O., Merryfield, W.J.: 1996, Mon. Not. Roy. Astron. Soc., 278, 437.

Basu, S., Antia, H.M., Bogart, R.S.: 2004, Astrophys. J., 610, 1157.

Berrilli, F., Ermolli, I., Florio, A., Pietropaolo, E.: 1999, Astron. Astrophys., 344, 965.

Braun, D.C., Duvall, T.L.Jr., LaBonte, B.J.: 1987, Astrophys. J., 319, L27.

Burtseva, O., Kholikov, S., Serebryanskiy, A., Chou, D.-Y.: 2007, Solar Phys., 241, 17.

Chaplin, W.J., Elsworth, Y., Isaak, G.R., Miller, B.A., New, R.: 2000, Mon. Not. Roy. Astron. Soc., 313, 32.

Chen, K.-R., Chou, D.-Y., TON Team: 1996, Astrophys. J., 465, 985.

Chou, D.-Y., Ladenkov, O.: 2007, Solar Phys., 241, 7.

Chou, D.-Y., Chou, H.-Y., Hsieh, Y.-C., Chen, K.-R. et al.: 1996, Astrophys. J., 459, 792.

Chou, D.-Y., Serebryanskiy, A., Ye, Y.-J., Dai, D.-C., Khalikov, S.: 2001, Astrophys. J., 554, L229.

Donea, A.-C., Braun, D.C., Lindsey, C.: 1999, Astrophys. J., 513, L143.

Gavryusev, V.G., and Gavryuseva, E.A.: 1999, Mon. Not. Roy. Astron. Soc., 303, L63.

Haber, D.A., Toomre, J., Hill, F., Gough, D.O.: 1988, In Domingo, V., Rolfe, E.J. (eds.), Seismology of the Sun and Sun-Like Stars, ESA SP-286, Noordwijk, the Netherlands, 301.

Hagenaar, H.J., Schrijver, C.J., Title, A.M.: 2003, Astrophys. J., 584, 1107.

Hanslmeier, A., Muller, R.: 2002, In Wilson, A. (ed.), Solar variability: from core to outer frontiers. The 10th European Solar Physics Meeting, ESA SP-506, 2. ESA, Noordwijk, 843.

Harvey, J.: 1971, Publications of the Astronomical Society of the Pacific, 83, No.495, 539.

Harvey, J.W., Branston, D., Henney, C.J., Keller, C.U.: 2007, Astrophys. J., 659, L177.

Harvey, K.L.: 1994, In Pap, J.M., Frolich, C., Hudson, H.S., Solanki, S. (eds.), Proceedings of IAU Colloquium No.143: The Sun as a variable star. Solar and stellar irradiance variations, Cambridge: Cambridge Univ. Press, 217.

Hindman, B.W., Jain, R., Zweibel, E.G.: 1997, Astrophys. J., 476, 392.

Houdek, G., Chaplin, W.J., Appourchaux, T., Christensen-Dalsgaard, J., Dappen, W., Elsworth, Y., Gough, D.O., Isaak, G.R., New, R., Rabello-Soares, M.C.: 2001, Mon. Not. Roy. Astron. Soc., 327, 483.

Howe, R., Chaplin, W.J., Elsworth, Y.P., Hill, F., Komm, R.W., Isaak, G.R., New, R. : 2003, Astrophys. J., 588, 1204.

Howe, R., Komm, R.W., Hill, F., Haber, D.A., Hindman, B.W.: 2004, Astrophys. J., 608, 562.

Jackson, J.D.: 1975, Classical Electrodynamics (2nd edition), John Wiley, New York, 303.

Jimenez-Reyes, S.J., Garcia, R.A., Jimenez, A., Chaplin, W.J.: 2003, Astrophys. J., 595, 446.

Karoff, C., Kjeldsen, H.: 2008, Astrophys. J., 678, L73.

Komm, R., Howe, R., Hill, F.: 2000, Astrophys. J., 543, 472.

Komm, R., Howe, R., Hill, F.: 2002, Astrophys. J., 572, 663.

Kosovichev, A.G., Duvall, T.L.Jr.: 1997, In Pijpers, F.P., Christensen-Dalsgaard, J., Rosenthal, C.S. (eds.), SCORe'96: Solar Convection and Oscillations and their Relationship, Kluwer Academic Publishers, Dordrecht, Astrophysics and Space Science Library, 225, 241.

Kosovichev, A.G., Zharkova, V.V.: 1998, Nature, 393, 317.

Lin, H.: 1995, Astrophys. J., 446, 421.

Lites, B.W., Kubo, M., Socas-Navarro, H., Berger, T., Frank, Z., Shine, R., Tarbell, T., Title, A., Ichimoto, K., Katsukawa, Y., Tsuneta, S., Suematsu, Y., Shimizu, T., Nagata, S.: 2008, In Matthews, S.A., Davis, J.M., Harra, L.K. (eds.), First Results From Hinode, San Francisco: ASP Conference Series, 397, 17.

Martinez-Oliveros, J.C., Moradi, H., Donea, A.-C.: 2008, Solar Phys., 251, 613.

Meunier, N., Roudier, T., Rieutord, M.: 2008, Astron. Astrophys., 488, 1109.

Muller, R.: 1988, Adv. Space Res., 8, 159.

Muller, R., Hanslmeier, A., Saldana-Munoz, M.: 2007, Astron. Astrophys., 475, 717.

Parchevsky, K.V., Kosovichev, A.G.: 2007, Astrophys. J., 666, L53.

Pauluhn, A., Solanki, S.K.: 2003, Astron. Astrophys., 407, 359.

Pevtsov, A.A., Acton, L.W.: 2001, Astrophys. J., 554, 416.

Rajaguru, S.P., Basu, S., Antia, H.M.: 2001, Astrophys. J., 563, 410.

Salabert, D., Jimenez-Reyes, S.J.: 2006, Astrophys. J., 650, 451.

Saldana-Munoz, M., Muller, R., Hanslmeier, A.: 2004, Hvar Obs. Bull., 28, 9.

Sanchez Almeida, J.: 2003, Astron.Astrophys., 411, 615. 
Scherrer, P.H., Bogart, R.S., Bush, R.I., Hoeksema, J.T., Kosovichev, A.G., Schou, J., Rosenberg, W., Springer, L., Tarbell, T.D., Title, A., Wolfson, C.J., Zayer, I., MDI Engineering Team: 1995, Solar Phys., 162, 129.

Topka, K.P., Tarbell, T.D., Title, A.M.: 1997, Bull. Am. Astronom. Soc., 29, 904.

Wenzler, T., Solanki, S.K., Krivova, N.A., Fluri, D.M.: 2004, Astron.Astrophys., 427, 1031.

Woods, D.T., Cram, L.E.: 1981, Solar Phys., 69, 233. 
burtseva09_4.tex; 21/11/2018; 3:46; p. 14 